\title{
Realizations of Uncertain Systems and Formal Power Series
}

\author{
Carolyn Beck John Doyle* \\ Department of Electrical Engineering, M/S 116-81 \\ California Institute of Technology, Pasadena, CA 91125
}

\begin{abstract}
Rational functions of several noncommuting indeterminates arise naturally in robust control when studying systems with structured uncertainty. Linear fractional transformations (LFTs) provide a convenient way of obtaining realizations of such systems and a complete realization theory of LFTs is emerging. This paper establishes connections between a minimal LFT realization and minimal realizations of a formal power series, which have been studied extensively in a variety of disciplines. The result is a fairly complete generalization of standard minimal realization theory for linear systems to the formal power series and LFT setting.
\end{abstract}

\section{Introduction}

A convenient and general framework for representing and manipulating uncertain models is to use linear fractional transformations (LFTs) on structured perturbations. A comprehensive theory, which we will not review here, has been developed for such systems, involving a great variety of assumptions on the perturbations. The perturbations are often as sumed to be norm-bounded operators, and frequently have additional structure, such as time-invariant or real parametric. Generally speaking, the more structure that is imposed on the uncertainty, the more difficult computation becomes. If the only structure we assume for the uncertainty is spatial (block-diagonal), then an LFT reduces to a representation of rational functions in multiple noncommuting indeterminates, which we will refer to here as an LFT system. An LFT system may then be viewed as a particular realization of a formal power series (FPS) [4]. Formal power series have long been used in many branches of mathematics, for example in combinatorics and enumeration. More specifically, rational series have been shown to have a rich structure, and have been used extensively in theoretical computer science for the development of automata and formal language theory. In this paper we will focus on realization theory for LFT systems and their relationship with both standard state-space realization theory and formal power series representations of nonlinear systems.

For state-space realizations of rational functions involving only a single indeterminate there are standard tests for minimality in terms of controllability and observability, which can in turn be characterized by rank tests on gramians, solutions to Lyapunov equations. We generalize this to LFT realizations for uncertain systems, first by showing that if there exist singular solutions to either of a pair of Lyapunov inequalities, in the form of linear matrix inequalities (LMIs), then the LFT representation is reducible. Conversely, we also show that if a smaller dimension model for an LFT realization exists for which there is no error between representations, then singular solutions to the same Lyapunov inequalities exist. Finally, all irreducible, or minimal, LFT realizations have the same dimension, differ by an allowable similarity transformation, and any realization can be reduced to a minimal one by similarity transformation and truncation.

We relate the LFT realizations we obtain to the well-known results on realizations of formal power series (FPS) originating from the work of Fliess (see [7], [8] and the references therein), and Isidori [11], who used rational series to develop a realization theory for bilinear systems. Sontag also used such series for a realization theory of discrete time nonlinear systems [16]. The realizations for bilinear and nonlinear systems,

\footnotetext{
*also Control and Dynamical Systems
}

which we will refer to as rational realizations, have a different form than LFT realizations. In this paper we establish a fairly complete connection between LFTs and rational realizations. Using these connections we obtain the same necessary and sufficient condition for reducibility of LFT system realizations as in [1] via a much simpler argument. Although connections with other work on formal power series are not directly pursued here, there exists a well-developed realization theory for more general rational series to which it appears further connections can be made. This will be elaborated upon in a future paper [2].

This work is motivated by earlier work on the generalization of balanced truncation model reduction methods ([6], [9], [10], [12]) to uncertain and multi-dimensional systems, presented in [3]. These model reduction results lead naturally to the development of a notion of minimality for uncertain systems, presented recently in [1].

We review relevant standard realization theory results, provide background material for formal power series and LFTs, and state our necessary and sufficient reducibility condition in Section 2. Connections between rational series and LFT realizations are discussed in Section 3. In Section 4 the reducibility result is discussed and proofs for the necessity condition provided.

\section{Background}

The notation we use is as follows: we represent complex and real matrices by $\mathbb{C}^{n \times m}$ and $\mathbb{R}^{n \times m}$, and the integers by $\mathbb{Z}$. The maximum singular value of $A \in \mathbb{C}^{n \times m}$ is denoted by $\bar{\sigma}(A)$, and $A^{*}$ denotes the Hermitian conjugate. The identity matrix is denoted by $I$. The dimensions of a matrix $A$ are denoted $\operatorname{dim}(A), l_{2}$ denotes the space of sequences which are square surnmable, and $\mathcal{L}\left(l_{2}\right)$ represents the space of all linear timevarying operators on $l_{2}$; the shift operator on $l_{2}$ is denoted by $z^{-1}$. For notational convenience, spatial dimensions will be given only when required for clarity of the discussion.

\subsection{Standard Realization Theory}

We review a few standard results from realization theory for one-dimensional (1D) discrete time systems with no uncertainty. Suppose $M=\left[\begin{array}{ll}A & B \\ C & D\end{array}\right]$ is a stable system realization (i.e., $\rho(A)<1$ ) for a system with transfer function $G\left(z^{-1}\right)=$ $D+C z^{-1}\left(I-A z^{-1}\right)^{-1} B$, and $Y$ and $X$ are the controllability and observability gramians, respectively. That is, $Y=Y^{*} \geq 0$ and $X=X^{*} \geq 0$ satisfy the Lyapunov equations

$$
\begin{aligned}
& A Y A^{*}-Y+B B^{*}=0 \\
& A^{*} X A-X+C^{*} C=0 .
\end{aligned}
$$

From standard Lyapunov theory, we know that the pair $(A, B)$ is controllable if and only if $Y>0$, and $(C, A)$ is observable if and only if $X>0$, in which case we know the realization is also minimal, or irreducible. An equivalent condition for controllability and observability is that the ranks of both the controllability matrix $C$ and observability matrix $\mathcal{O}$ are full rank.

If the realization, $M$, of a ID system is not minimal, then there exists a similarity transformation, $T$, such that the controllability and observability gramians are diagonal, and the controllable and observable subsystem is balanced. The following theorem is standard, so the proof is omitted. 
Theorem 1 For any stable system realization $M=\left[\begin{array}{ll}A & B \\ C & D\end{array}\right]$ there exists $T$ such that $\widehat{M}=\left[\begin{array}{cc}T A T^{-1} & T B \\ C T^{-1} & D\end{array}\right]$ has controllability and observability gramians given by

$$
Y=\left[\begin{array}{cccc}
\Sigma_{1} & & & \\
& \Sigma_{2} & & \\
& & 0 & \\
& & & 0
\end{array}\right] \text { and } X=\left[\begin{array}{llll}
\Sigma_{1} & & & \\
& 0 & & \\
& & \Sigma_{3} & \\
& & & 0
\end{array}\right]
$$

respectively, with $\Sigma_{1}, \Sigma_{2}, \Sigma_{3}$ diagonal and positive definite.

Since the uncontrollable and unobservable modes of any system realization are not present in the corresponding system transfer function we can truncate the associated states, corresponding to the zeros in $Y$ and $X$ above, and obtain a minimal realization which has both gramians equal to $\Sigma_{1}$. Such a system is reducible in that there exists a lower order equivalent realization. In this paper, we show that the same is true for uncertain systems by generalizing the use of similarity transformations and truncations. We first present a brief summary of relevant results for formal power series.

\subsection{Formal Power Series}

In order to relate LFTs to formal power series, we first review basic notation for these series and several well-known results ([11], [4]). In the most general form, a formal power series $S$ is a function $X^{*} \rightarrow \mathcal{R}$, defined by

$$
S=\sum_{w \in X^{*}} s(w) w
$$

where $\chi^{*}$ is the free monoid generated by a finite nonempty set $X$, and $\mathcal{R}$ is a semiring. An element, $w$, of $X^{*}$ is called a word, where the length of a word $w=x_{1} x_{2} \cdots x_{n}$ is $\mathrm{n}$, and is denoted by $|w|$, and the empty word is denoted by 1 . A coefficient of the series is denoted by $s(w)$, and is the image by $S$ of a word $w$, that is, $s(w) \in \mathcal{R}$. The product operation of the monoid $x^{*}$ is the concatenation defined by $\left(x_{1} \cdots x_{n}\right)$. $\left(y_{1} \cdots y_{p}\right)=x_{1} \cdots x_{n} y_{1} \cdots y_{p}$.

Definition 1 The Hankel matrix of a formal series $S$ is the matrix $\mathcal{H}_{S}$ indexed by $X^{*} \times X^{*}$ defined by $\mathcal{H}_{S}(u, v)=s(u \cdot v)$, for all words $u, v \in X^{*}$.

A standard result for formal series connects the rank of the Hankel matrix with the rank of the series, the definition of which for the most general power series is beyond the scope of this paper. However, for rational series, it is well-known that there exist linear representations of $S$, and that the rank of the series is equal to the minimal dimension of all such representations. Furthermore, a given series is rational if and only if the rank of it's Hankel matrix is finite (see [4] and the references therein for details).

We consider only rational, or finite rank series in this paper. In keeping with the notation used for rational series in the development of nonlinear realization theory, to which we will draw the most explicit connections, we consider the set of $p$ noncommuting variables, $x=\left\{\delta_{1}, \ldots, \delta_{p}\right\}$, and the associated index set $\mathcal{I}=\{1,2, \ldots, p\}$. Let $I_{k}$ denote the set of all sequences of $k$ elements $\left(i_{k} \ldots i_{1}\right)$ of 7 , where the empty sequence is denoted by $\varnothing$. Define $1^{*}=\bigcup_{k>0} 1_{k}$, where $I^{*}$ has the composition rule, $\left(i_{k} \ldots i_{1}\right)\left(j_{l} \ldots j_{1}\right) \mapsto\left(i_{k} \ldots i_{1} j_{l} \ldots j_{1}\right)$, and $I_{0}=\varnothing$. Then, to each multi-index $\left(i_{k} \ldots i_{0}\right)$ we associate the word $\left(\delta_{i_{k}} \cdots \delta_{i_{0}}\right)$. As in [11], we consider a forma] power series in $p$ noncommutative indeterminates with coefficients in the ring of real matrices, $\mathbb{R}^{m \times q}$, that is, the mapping $S: I^{*} \rightarrow \mathbb{R}^{m \times q}$ represented by the form

$$
s=s(\varnothing)+\sum_{k=0}^{\infty} \sum_{i_{0}, \ldots, i_{k}=0}^{p} s\left(i_{k} \cdots i_{0}\right) \delta_{i_{k}} \cdots \delta_{i_{0}}
$$

where $s\left(i_{k} \cdots i_{0}\right)$ is the coefficient of the $\left(i_{k}, \ldots, i_{0}\right)$-th term. The Hankel matrix associated with this series is the infinite matrix whose elements are defined by $\mathcal{H}_{S}\left(i_{k} \ldots i_{0}, j_{l} \ldots j_{0}\right)=$ $s\left(i_{k} \cdots i_{0} j_{l} \cdots j_{0}\right)$.
Given a rational series in $p$ indeterminants, constructive procedures for obtaining a specific form of linear representations exist [11], [16], which we will refer to as rational realizations, and denote by $\left\{H, M_{i}, G\right\}$, where

$$
S=H\left(I-\sum_{i=1}^{p} M_{i} \delta_{i}\right)^{-1} G=H \sum_{k=0}^{\infty}\left(\sum_{i=1}^{p} M_{i} \delta_{i}\right)^{k} G .
$$

Additionally, this realization is minimal, that is, $\operatorname{dim}(I)$ in the $\left(I-\sum_{i=1}^{p} M_{i} \delta_{i}\right)$ term is smallest among all possible rational realizations. This is the only dimension that can differ between the different rational realizations of the same series, and the dimension of a minimal rational realization is equal to $\operatorname{rank}\left(\mathcal{H}_{S}\right)$ [4]. Note that the rank as defined for the FPS Hankel matrix does not reduce to the the rank of the Hankel operator as it is usually defined for 1D systems. This is discussed in more detail in Section 3

Alternatively, if one is given a realization $\left\{H, M_{i}, G\right\}$ for rational series, this realization is minimal if the matrices $\mathcal{O}_{S}$ and $C_{S}$ defined by

$\mathcal{O}_{S}\left(i_{k} \ldots i_{0}\right)=H M_{i_{k}} \cdots M_{i_{0}}$ and $C_{S}\left(j_{l} \ldots j_{0}\right)=M_{j_{l}} \cdots M_{j_{0}} G$ are both full rank [17], since $\mathcal{H}_{S}=\mathcal{O}_{S} C_{S}$ and it is readily seen that $\operatorname{rank}\left(\mathcal{H}_{S}\right)=\min \left(\operatorname{rank}\left(\mathcal{O}_{S}\right), \operatorname{rank}\left(C_{S}\right)\right)$. Furthermore, the rank test for $\mathcal{O}_{S}$ and $C_{S}$ can be performed on finite matrjces [17].

\subsection{Linear Fractional Transformations}

The LFT paradigm, defined below and pictured in Figure 1, allows for a mathematical representation for uncertainty in system models. The source of such uncertainty might be combinations of unknown nonlinearities, unmodelled dynamics, external disturbances, and parameter variations.

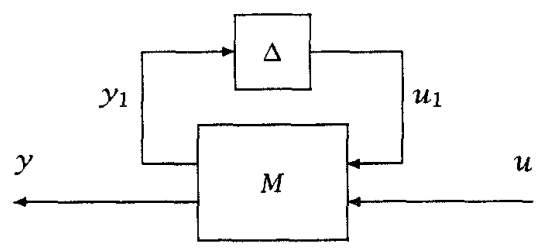

Figure 1: MD/Uncertain System

We assume $M=\left[\begin{array}{cc}A & B \\ C & D\end{array}\right]$ is a given system realization matrix, that is, what is known about the system, and $\Delta$ represents the system uncertainty, namely, what is unknown or poorly understood about the system, or possibly components of the system which are not time-invariant. We assume $\Delta$ lies in some prescribed set, and one uncertainty set $\Delta$ that is widely considered is

$$
\Delta=\left\{\operatorname{diag}\left[\delta_{1} I_{n_{1}}, \ldots, \delta_{p} I_{n_{p}}\right]: \delta_{i} \in \mathcal{L}\left(l_{2}\right)\right\} .
$$

This set is relatively unstructured, and leads to the strongest analysis results. For analysis purposes, we often consider $\Delta$ which lie in a norm-bounded subset of $\boldsymbol{\Delta}$, for example,

$$
\mathbf{B} \Delta=\left\{\Delta \in \Delta:\|\Delta\|_{l_{2} \rightarrow l_{2}} \leq 1\right\},
$$

where $\|\cdot\|_{l_{2} \rightarrow l_{2}}$ denotes the induced norm. Note that $\delta_{i} \in \mathcal{L}\left(l_{2}\right)$ allows time-varying operators on $l_{2}$, which are not commutative.

The input/output mapping for the system denoted by Figure 1 is given by the LFT

$$
y=(\Delta \star M) u=\left(D+C \Delta(I-A \Delta)^{-1} B\right) u, \Delta \in \Delta
$$

whenever the inverse is well-defined. We will denote these uncertain system models by the pair $(\Delta, M)$. For example, 1D systems with no uncertainty may be represented by a LFT on $z^{-1}$, that is, let $\Delta=z^{-1} I$, then $\Delta \star M=D+C(z I-A)^{-1} B$. Multi-dimensional systems are realized in this framework in a similar fashion. 
We say that the uncertain system represented by a LFT on a $\Delta$ structure is stable when the I/O map $(\Delta \star M)$ is well-defined for every $\Delta \in \mathbf{B} \Delta$; for $\Delta \subset \mathcal{L}\left(l_{2}\right),(\Delta \star M)$ is well-defined as a map on signals in $l_{2}$ when $(I-A \Delta)$ is invertible as an operator on $l_{2}$. In particular, when all $\delta_{i} \in \mathcal{L}\left(l_{2}\right)$ are time-varying (with the possible exception of one transform variable), then an uncertain system $(\Delta, M)$ is stable for all $\Delta \in \mathbf{B} \Delta$ if and only if there exists a matrix $Y>0, Y \in \mathcal{T}^{\prime}$ such that

$$
A Y A^{*}-Y<0,
$$

where $\mathcal{T}=\{T: T \Delta=\Delta T, \forall \Delta \in \Delta\}$ is the commutator set for $\Delta([14]$, [13], [15]). Clearly an equivalent condition would be the existence of $X>0, X \in \mathcal{T}$ satisfying $A^{*} X A-X<$ 0 . By scaling such $Y$ and $X$ one can immediately deduce the following lemma.

Lemma 1 If $(\Delta, M)$ is stable, then there exist $Y \geq 0$ and $X \geq 0$, both in $\mathcal{T}$, which satisfy the Lyapunov inequalities

$$
A Y A^{*}-Y+B B^{*} \leq 0 \text { and } A^{*} X A-X+C^{*} C \leq 0 .
$$

For an uncertain or multi-dimensional system realization, matrices $Y$ and $X$ satisfying (5) are referred to as generalized gramians. Clearly, these Lyapunov inequalities can have many solutions which may be computed using recent LMI optimization algorithms (see [5] and the references therein).

We present a condition for reducibility in terms of the realization matrices $A, B$ and $C$ from a LFT representation and solutions $X$ and $Y$ to the Lyapunov inequalities. However, we utilize a rational series representation of the input/output mapping $(\Delta \star M)$ to construct the proof for this reducibility condition. This result, stated below, is proven in Section 4 . It is implicitly assumed that the reduced realization, $\left(\Delta_{r}, M_{r}\right)$, referred to in this theorem has input/output dimensions which are compatible with those of the original realization $(\Delta, M)$. The uncertainty structures we refer to are:

$$
\Delta=\left\{\operatorname{diag}\left[\delta_{1} I_{n_{1}}, \delta_{2} I_{n_{2}}, \ldots, \delta_{p} I_{n_{p}}\right]\right\}
$$

and

$$
\Delta_{r}=\left\{\operatorname{diag}\left[\delta_{1} I_{r_{1}}, \delta_{2} I_{r_{2}}, \ldots, \delta_{p} I_{r_{p}}\right]\right\}
$$

where $n_{i} \geq r_{i}$ for every $i=1, \ldots, p$ and the $\delta_{i}$ are noncommuting. For convenience we denote

$$
\tilde{\Delta}=\left\{\left[\begin{array}{cc}
\Delta & 0 \\
0 & \Delta_{r}
\end{array}\right]: \Delta \in \Delta_{,} \Delta_{r} \in \Delta_{r}\right\}
$$

Theorem 2 Suppose the stable system realization $M=\left[\begin{array}{cc}A & B \\ C & D\end{array}\right]$ and uncertainty set $\Delta$ as defined in (2) are given. There exists a reduced realization $M_{r}=\left[\begin{array}{cc}A_{r} & B_{r} \\ C_{r} & D\end{array}\right]$ and uncertainty set $\Delta_{r}$ such that $(\Delta \star M)-\left(\Delta_{r} \star M_{r}\right)=0$ for all $\tilde{\Delta} \in \mathbf{B} \tilde{\Delta}$ if and only if there exists singular $X \geq 0$ or $Y \geq 0$, both in $\mathcal{T}$, satisfying

(i) $A Y A^{*}-Y+B B^{*} \leq 0$

or

(ii) $A^{*} X A-X+C^{*} C \leq 0$

One notable resuit which follows immediately from the proof for this theorem is that all minimal realizations for an uncertain system may be obtained by similarity transformations and truncations, as in the standard case, where now the allowed similarity transformations are restricted to the set $\mathcal{T}$. This result is stated in the following corollary.

Corollary 1 Given a stable system realization $(\Delta, M)$, all minimal realizations are given by similarity transformations,

$$
\widehat{M}=\left[\begin{array}{cc}
T A T^{-1} & T B \\
C T^{-1} & D
\end{array}\right]: T \in \mathcal{T},
$$

and truncations.

In particular, $\Delta \star M=\Delta \star \widehat{M}$ for all $\Delta \in \Delta$ and $T \in \mathcal{T}$.

If we consider the simplest case for these LFT representations of uncertain systems, i.e., there is no uncertainty and $\Delta=z^{-1} I$, we obtain the standard results, excepting the inequalities in the Lyapunov equations. At the other extreme, in the case of formal power series, we do not even have the operator structure for $\Delta$, as the $\delta_{i}$ are simply noncommuting indeterminates. Thus stability and norms have no meaning, and indeed are artificial in the context of realization theory. There are many ways to extend the LFT machinery to this case, but the simplest way to remove the stability requirements for the $A$ matrix is by scaling. Note that there will always be some value $\gamma>0$ sufficiently small (e.g. $\gamma<1 /\|A\|$ ) such that there exists a matrix $Y>0, Y \in \mathcal{T}$ satisfying

$$
\gamma^{2} A Y A^{*}-Y<0 .
$$

In this case, the $\mathrm{I} / \mathrm{O}$ map $(\Delta \star M)$ is well-defined on $l_{2}$ for every $\Delta \in \mathbf{B}_{y} \Delta=\left\{\Delta \in \Delta:\|\Delta\|_{l_{2} \rightarrow l_{2}} \leq y\right\}$, and solutions $Y \geq 0$ and $X \geq 0$ in the commutator set $\mathcal{T}$ can be found satisfying the LMIs

$$
\gamma^{2} A Y A^{*}-Y+B B^{*} \leq 0 \text { and } \gamma^{2} A^{*} X A-X+C^{*} C \leq 0 .
$$

If singular $X$ and $Y$ can be found, the realization is then reducible, as in Theorem 2, with respect to the uncertainty set $B_{\gamma} \Delta$, and further reducing the value of $\gamma$ does not effect the existence of singular solutions to these LMIs. For convenience, we will henceforth assume that all LFT realizations we consider satisfy the LMI in (6) with $\gamma=1$, as otherwise all results hold for realizations and uncertainty sets scaled by some positive $\gamma<1$.

Efficient convex optimization algorithms have been developed for solving LMI problems. The fact that we would like to find singular solutions to these LMIs complicates the computational requirements, resulting in a non-convex optimization problem. However, in the case of non-singular $X$ and $Y$, we can still reduce LFT realizations with guaranteed error bounds [3]. Furthermore, as we will see in the next section, we can relate minimality of LFT realizations to minimality of corresponding rational realizations, giving an alternative method for finding minimal realizations.

\section{LFT and Rational Realizations: Connec- tions}

Given a system realization, $(\Delta, M)$, with corresponding $1 / O$ behav<iour described by the LFT, $D+C \Delta(I-A \Delta)^{-1} B$, we can form a power series by expanding the $(I-A \Delta)^{-1}$ term, giving

$$
S=\Delta \star M=D+\sum_{k=0}^{\infty} C \Delta(A \Delta)^{k} B .
$$

To obtain a relation between a LFT realization and a minimal rational realization, $\left\{H, M_{i}, G\right\}$, we compare the terms in the respective series defined by these realizations. We first partition the matrices $A, B$, and $C$ conformally with the $\Delta$ structure, that is,

$$
A=\left[\begin{array}{ccc}
A_{11} & \cdots & A_{1 p} \\
\vdots & \ddots & \vdots \\
A_{p 1} & \cdots & A_{p p}
\end{array}\right] ; \quad B=\left[\begin{array}{c}
B_{1} \\
\vdots \\
B_{p}
\end{array}\right] ; \quad C=\left[\begin{array}{llll}
C_{1} & \cdots & C_{p}
\end{array}\right] .
$$

Then, expanding the series $S$ defined in (7) gives

$$
\begin{gathered}
\Delta \star M=D+\sum_{i=1}^{p} C_{i} B_{i} \delta_{i+} \\
\sum_{k=0}^{\infty} \sum_{i_{0}, \ldots, i_{k}=1}^{p} C_{i_{k}} A_{i_{k} i_{k-1}} \cdots A_{i_{1} i_{0}} B_{i_{0}} \delta_{i_{k}} \cdots \delta_{l_{0}} .
\end{gathered}
$$

As discussed in Section 2.2, we can compute a minimal realization $\left\{H, M_{i}, G\right\}$ for a given series $S_{\text {, such that }}$

$$
S=H \sum_{k=0}^{\infty}\left(\sum_{i=1}^{p} M_{i} \delta_{i}\right)^{k} G .
$$

Suppose we start with this minimal rational realization $\left\{H, M_{i}, G\right\}$ and factor $M_{i}=L_{i} R_{i}$, where $L_{i}$ has full column rank and $R_{i}$ has full row rank. This factorization is nonunique; we will henceforth refer to such factorizations of a matrix as minimal rank factorizations. By equating terms in (7) and (8) we 
obtain an LFT representation from the rational series representation:

$$
D=H G, C_{i}=H L_{i}, B_{i}=R_{i} G \text {, and } A_{i j}=R_{i} L_{j} .
$$

It is readily seen that $\operatorname{dim}\left(A_{i i}\right)=\operatorname{rank}\left(M_{i}\right)$

Conversely, if we are given a minimal LFT realization, we may obtain a corresponding rational realization, $\left\{H, M_{i}, G\right\}$, by computing a minimal rank factorization

$$
\left[\begin{array}{ll}
A & B \\
C & D
\end{array}\right]=\left[\begin{array}{l}
R \\
H
\end{array}\right][L \quad G]
$$

where $\left[\begin{array}{c}R \\ H\end{array}\right]$ has full column rank, and $\left[\begin{array}{ll}L & G\end{array}\right]$ has full row rank, and $R$ and $L$ are partitioned into $p$ submatrices, e.g, $L=\left[L_{1} L_{2} \cdots L_{p}\right]$. Defining $M_{i}=L_{i} R_{i}$ gives the realization, $\left\{H, M_{i}, G\right\}$

Up to an allowable similarity transformation (which accounts for the nonuniqueness), the conversion procedure of (10) is clearly reversible, but the minimal dimension is $\operatorname{dim}(A)$ for LFT realizations and $\operatorname{dim}\left(M_{i}\right)=\operatorname{rank}\left(\mathcal{H}_{S}\right)$ for rational realizations. These two dimensions are in general not equal, with the dimensions of LFT realizations being related to the $\operatorname{rank}\left(M_{i}\right)$ of the rational realizations. Several bounds can be constructed to relate these dimensions, but neither dimension is always greater than or equal to the other. For uncertain systems the $\operatorname{dim}(A)$ is more natural since it measures the number of copies of the $\delta_{i}$ required to build an interconnection that realizes the series, a generalization of the number of delays or integrators needed to realize a $1 D$ transfer function. Furthermore, the $\operatorname{rank}\left(\mathcal{H}_{S}\right)$ de pends on the constant term $s(\varnothing)=H G=D$, whereas $\operatorname{dim}(A)$ clearly does not. We could allow an additional constant term in the rational realization so that the $\operatorname{rank}\left(\mathcal{H}_{S}\right)$ is minimized, then the dimension of the "minimal " realization $\left\{H, M_{i}, G\right\}$ is also minimized.

The deeper question is whether the above formulas trans form one type of minimal realization into the other. The an swer is affirmative. By Corollary 1 , all minimal I.FT realizations can be obtained from equivalent LFT realizations by similarity transformation and truncation, operations which necessarily do not increase rank. Thus, $\operatorname{rank}\left(\left[\begin{array}{cc}A & B \\ C & D\end{array}\right]\right)$ is minimized for a minimal LFT realization. As a result, computing the factorization in (10) for a minimal LFT realization yields a minimal $\left\{H, M_{i}, G\right\}$ realization, and moreover,

$$
\operatorname{rank}\left(H_{S}\right)=\operatorname{rank}\left(\left[\begin{array}{cc}
A & B \\
C & D
\end{array}\right]\right) .
$$

The converse result, that of obtaining a minimal LFT realization from a minimal rational realization, can be shown using the well-known result for rational series that all minimal linear representations are similar [7]. This will be discussed in more detail in [2].

\section{Reducibility Conditions for LFTs}

We present a generalization of the role that Lyapunov equations and gramians play in the quantification of minimality for standard systems to LFT systems represented by the pair $(\Delta, M)$. This result was first stated and proved in [1] for systems mapping $l_{2}$ signals to $l_{2}$ signals, and with uncertainty represented by arbitrary time-varying operators on $l_{2}$. In this section, we prove the necessity condition using a rational series representation, which results in a much simpler proof than that given in [1]. For completeness, we state the sufficiency condition below in Theorem 3. We assume throughout this section that the LMI condition in (4) is satisfiable, again noting that this is always true up to a scaling factor $\gamma$.

Theorem 3 [I] (Sufficiency) Suppose the system realization $M=\left[\begin{array}{ll}A & B \\ C & D\end{array}\right]$ with uncertainty structure $\Delta$ is given. Then there exists a reduced realization $M_{r}=\left[\begin{array}{cc}A_{r} & B_{r} \\ C_{r} & D\end{array}\right]$ and uncertainty structure $\Delta_{r}$ such that $(\Delta \star M)-\left(\Delta_{r} \star M_{r}\right)=0$ for all $\tilde{\Delta} \in \mathrm{B} \tilde{\Delta}$ if there exists singular $X \geq 0$ or $Y \geq 0$, both in $\mathcal{T}$, satisfying (i) $A Y A^{*}-Y^{*}+B B^{*} \leq 0$

or

(ii) $A^{*} X A-X+C^{*} C \leq 0$

This sufficiency condition holds when the $\delta_{i}$ are defined as transform variables, norm-bounded perturbations, timevarying operators on $l_{2}$, or any combination. The proof for Theorem 3 reveals that if singular generalized gramians exist, then the system realization can be (allowably) transformed to a realization having an uncontrollable/unobservable like decomposition.

The corresponding necessity condition is proven, via the following two lemmas, for the case of noncommuting $\delta_{i}$, for example, time-varying operators.

Lemma 2 Given the system realization $M=\left[\begin{array}{ll}A & B \\ C & 0\end{array}\right]$, and uncertainty structure $\Delta$ where the $\delta_{i}, i=1, \ldots, p$, are noncom muting variables, then, if $(\Delta \star M)=0$ for all $\Delta \in \mathbf{B} \Delta$, there exists $X \geq 0$ and $Y \geq 0$, both in $\mathcal{T}$, satisfying

(i) $A Y A^{*}-Y+B B^{*} \leq 0$

(ii) $A^{*} X A-X+C^{*} C \leq 0$

(iii) $X Y=0$

Proof. By assumption, $\Delta \star M=\sum_{k=0}^{\infty} C \Delta(A \Delta)^{k} B=0$. This series is identically zero if and only if each coefficient in the series is zero, that is,

$$
C_{i} B_{i}=0, C_{i} A_{i j} B_{j}=0, C_{i} A_{i j} A_{j k} B_{k}=0, \ldots,
$$

for every $i, j, k=1, \ldots, p$. We consider each set of terms separately and show that the given realization may be allowably transformed to one having an uncontrollable/unobservablelike decomposition. First, consider the $C_{i} B_{i}$ terms:

$$
\begin{gathered}
C_{i} B_{i}=0 \Longleftrightarrow \exists T_{i} \text { nonsingular, such that } \\
C_{i} T_{i}^{-1}=\left[\begin{array}{llll}
0 & 0 & \widetilde{C}
\end{array}\right]_{i} \text { and } T_{i} B_{i}=\left[\begin{array}{l}
\widetilde{B} \\
0 \\
0
\end{array}\right]_{i},
\end{gathered}
$$

where $\widetilde{C}_{i}$ has full column rank, $\widetilde{B}_{j}$ has full row rank, and the submatrices of $C_{i}$ and $B_{i}$ are conformally partitioned, for each $i, j=1, \ldots, p$. We will henceforth absorb all such transformations and assume the realization matrices are already structured into zero and non-zero block submatrices. Additionally, we partition $A_{i j}$ conformally with $C_{i}$ and $B_{j}$.

Now consider the $C_{i} A_{i j} B_{j}$ terms:

$$
\begin{aligned}
C_{i} A_{i j} B_{j} & =\left[\begin{array}{lll}
0 & 0 & \widetilde{C}
\end{array}\right]_{i}\left[\begin{array}{lll}
A^{11} & A^{12} & A^{13} \\
A^{21} & A^{22} & A^{23} \\
A^{31} & A^{32} & A^{33}
\end{array}\right]_{i j}\left[\begin{array}{l}
\tilde{B} \\
0 \\
0
\end{array}\right]_{j}=0 \\
& \Leftrightarrow \widetilde{C}_{i} A_{i j}^{31} \widetilde{B}_{j}=0 \Leftrightarrow A_{i j}^{31}=0
\end{aligned}
$$

since $\widetilde{C}_{i}$ and $\widetilde{B}_{j}$ are full rank.

Next consider the $C_{i} A_{i j} A_{j l} B_{l}$ terms:

$C_{i} A_{i j} A_{j l} B_{l}=\left[\begin{array}{lll}0 & \widetilde{C}_{i} A_{i j}^{32} & \widetilde{C}_{i} A_{i j}^{33}\end{array}\right]\left[\begin{array}{c}A_{j l}^{11} \widetilde{B}_{l} \\ A_{j l}^{21} \widetilde{B}_{l} \\ 0\end{array}\right]=0 \Longleftrightarrow A_{i j}^{32} A_{j l}^{21}=0$.

As with the $C_{i} B_{i}$ terms, we can write $A_{i j}^{32}=\left[\begin{array}{lll}0 & 0 & \widetilde{A}_{i j}^{32}\end{array}\right]$ and $A_{j l}^{21}=\left[\begin{array}{c}\tilde{A}_{j l}^{21} \\ 0 \\ 0\end{array}\right]$ where $\tilde{A}_{i j}^{32}$ has full column rank and $\tilde{A}_{j l}^{21}$ has full row rank.

We similarly evaluate a finite number of terms in the series, depending only on the dimensions of the realization matrices, resulting in an uncontrollable/unobservable-like decomposition structure for each $C_{j}, A_{i j}, B_{i}$ subsystem. This decom- 
position is generally written as follows:

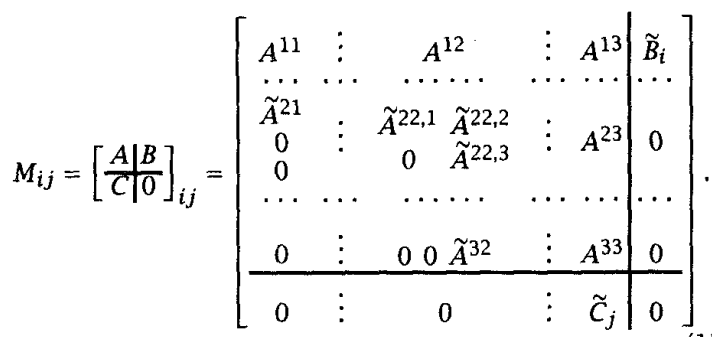

We can construct $\tilde{X}=\operatorname{diag}\left[0, \tilde{X}_{1}^{22}, 0, \tilde{X}_{2}^{22}, \ldots, 0, \tilde{X}_{p}^{22}\right] \geq 0$ and $\tilde{Y}=\operatorname{diag}\left[\tilde{Y}_{1}^{11}, 0, \tilde{Y}_{2}^{11}, 0, \ldots, \tilde{Y}_{p}^{11}, 0\right] \geq 0$, satisfying the Lyapunov inequalities, where $\tilde{X} \geq 0$ and $\tilde{Y} \geq 0$, both in $\mathcal{T}$, are partitioned according to the decompositions shown in (13).

Specifically, consider the largest dimensioned lower left submatrix of $A_{i i}$ which is identically zero. Denote the number of columns of $A_{i i}$ to the right of this zero block by $\tilde{n}_{i}$, and the number of rows above the zero block by $\widetilde{m}_{i}$. By assumption, there exist $Y=\operatorname{diag}\left[Y_{1}, Y_{2}, \ldots, Y_{p}\right]>0$ and $X=$ $\operatorname{diag}\left[X_{1}, X_{2}, \ldots, X_{p}\right]>0$, both in $\mathcal{T}$, satisfying $A Y A^{*}-Y<0$ and $A^{*} X A-X<0$. Partition each $X_{i}$ and $Y_{i}$ as follows:

$$
X_{i}=\left[\begin{array}{cc}
X^{11} & X^{12} \\
\left(X^{12}\right)^{*} & \tilde{X}^{22}
\end{array}\right]_{i} \text { and } Y_{i}=\left[\begin{array}{cc}
\tilde{Y}^{11} & Y^{12} \\
\left(Y^{12}\right)^{*} & Y^{22}
\end{array}\right]_{i},
$$

where $\operatorname{dim}\left(\tilde{X}_{i}^{22}\right)=\tilde{n}_{i} \times \tilde{n}_{i}$ and $\operatorname{dim}\left(\tilde{Y}_{i}^{11}\right)=\widetilde{m}_{i} \times \widetilde{m}_{i}$. Applying block permutations to the above LMIs and scaling $\tilde{Y}$ and $\tilde{X}$ as necessary, it can be shown after some routine manipulations that conditions $(i)$ and $(i i)$ are satisfied, and clearly $\tilde{X} \tilde{Y}=0$. $\square$

In the proof of Lemma 2, it is implicitly assumed that the uncertainty structure is $\Delta=\left\{\operatorname{diag}\left[\delta_{1} I_{n_{1}}, \ldots, \delta_{p} I_{n_{p}}\right]\right\}$. The result can immediately be extended to uncertainty structures such as $\tilde{\Delta}=\left\{\operatorname{diag}\left[\delta_{1} I_{n_{1}}, \ldots, \delta_{p} I_{n_{p}}, \delta_{1} I_{r_{1}}, \ldots, \delta_{p} I_{r_{p}}\right]\right\}$ by permuting the subblocks of $\tilde{\Delta}$ and the system realization matrices.

Lemma 3 ([1]) Suppose $\tilde{X}=\left[\begin{array}{cc}X & X_{1} \\ X_{1}^{*} & X_{2}\end{array}\right] \geq 0$ and $\tilde{Y}=$ $\left[\begin{array}{cc}Y & Y_{1} \\ Y_{1}^{*} & Y_{2}\end{array}\right] \geq 0$, with $\operatorname{dim}(X)=\operatorname{dim}(Y)>\operatorname{dim}\left(X_{2}\right)=\operatorname{dim}\left(Y_{2}\right)$. If $\tilde{X} \tilde{Y}=0$, then either $X$ or $Y$ is singular.

Theorem 4 (Necessity): Suppose the system realization $M=$ $\left[\begin{array}{ll}A & B \\ C & D\end{array}\right]$ and uncertainty structure $\Delta$ as defined in (2) are given. If there exists a reduced realization $M_{r}=\left[\begin{array}{cc}A_{r} & B_{r} \\ C_{r} & D\end{array}\right]$ and uncertainty structure $\Delta_{r}$ such that $(\Delta \star M)-\left(\Delta_{r} \star M_{r}\right)=0$, for all $\tilde{\Delta} \in \mathbf{B} \tilde{\Delta}$, then there exists singular $X \geq 0$ or $Y \geq 0$, both in $\mathcal{T}$, satisfying

(i) $A Y A^{*}-Y+B B^{*} \leq 0$

or

(ii) $A^{*} X A-X+C^{*} C \leq 0$

Proof. Consider the difference system $(\tilde{\Delta} \star \widetilde{E})=(\Delta \star M)-$ $\left(\Delta_{r} \star M_{r}\right)$, that is $\widetilde{E}=\left[\begin{array}{ccc}A & 0 & B \\ 0 & A_{r} & -B_{r} \\ C & C_{r} & 0\end{array}\right]$ and $\widetilde{\Delta}=\left[\begin{array}{cc}\Delta & 0 \\ 0 & \Delta_{r}\end{array}\right]$. By Lemma 2 , if $(\widetilde{\Delta} \star \widetilde{E})=0$ then there exist $\tilde{X} \geq 0$ and $\tilde{Y} \geq 0$, both in $\widetilde{\mathcal{T}}$ satisfying the Lyapunov inequalities for the uncertain system $(\tilde{\Delta}, \widetilde{E})$, and $\tilde{X} \tilde{Y}=0$. Since $\widetilde{X}$ and $\tilde{Y}$ commute with $\widetilde{\Delta}$, they have the structure

$$
\tilde{X}=\left[\begin{array}{cc}
X & X_{1} \\
X_{1}^{*} & X_{2}
\end{array}\right] \text { and } \tilde{Y}=\left[\begin{array}{cc}
Y & Y_{1} \\
Y_{1}^{*} & Y_{2}
\end{array}\right]
$$

where $X$ and $Y$ commute with $\Delta$. Then $X \geq 0$ and $Y \geq 0$ satisfy the Lyapunov inequalities for the uncertain system $(\Delta, M)$, and by Lemma 3 either $X$ or $Y$ is singular.

\section{Acknowledgements}

The authors are extremely grateful to Eduardo Sontag for provocative and stimulating discussions, which motivated the results in this paper. We would also like to thank Allen Tannenbaum for suggesting that we consider formal power series and talk to Eduardo Sontag. This work was supported by AFOSR.

\section{References}

[1] Beck, C., 1994, "Minimality for Uncertain Systems and IQCs", Proceedings, 33rd CDC, Florida

[2] Beck, C., and Doyle, J.C., 1995, "Uncertain Systems and Formal Power Series", report in preparation

[3] Beck, C., Doyle, J.C., and Glover, K,. 1995, "Model Reduction of Multi-Dimensional and Uncertain Systems", submitted to IEEE Trans. on Automatic Control

[4] Berstel, J., and Reutenauer, C., 1988, "Rational Series and Their Languages", Monographs on Theoretical Computer Science, Springer-Verlag

[5] Boyd, S,. El Ghaoui, L., Feron, E., and Balakrishnan, V. 1994 "Linear Matrix Inequalities in Systems and Control Theory", SIAM

[6] Enns, D.F., 1984, Model Reduction for Control System Design, Ph.D. Dissertation, Stanford University.

[7] Fliess, M., 1974, "Matrices de Hankel", J. Math. pures et appl., 53, 197-222

[8] Fliess, M., Lamnabhi, M., and Lamnabhi, F., 1983, "An Algebraic Approach to Nonlinear Functional Expansions", IEEE Trans. on Circuits and Systems, Vol. 30, No. 8

[9] Glover, K., 1984, "All optimal Hankel-norm approximations of linear multivariable systems and their $L^{\infty}$-error bounds", Int. J. Control., Vol.39:1115-1193.

[10] Hinrichsen, D, and Pritchard, A.J., 1990, "An improved error estimate for reduced-order models of discrete-time systems", IEEE Trans. on Auto. Control, AC-35:317-320.

[11] Isidori, A., 1989 "Nonlinear Control Systems", SpringerVerlag

[12] B.C. Moore, 1981, "Principal Component Analysis of Linear Systems: Controllability, Observability and Model Reduction", IEEE Trans. Automat. Control, Vol. 26, 17-32.

[13] Megretskii, A., "Necessary and Sufficient Conditions of Stability: A Multiloop Generalization of the Circle Criterion", IEEE Trans., AC-38, No. 5, 1993

[14] Paganini, F., and Doyle, J., 1994, "Analysis of Implicit Uncertain Systems, Part I: Theoretical Framework", submitted to IEEE Trans. on Automat. Control

[15] Shamma, J., 1994, "Robust Stability with Time-Varying Structured Uncertainty", IEEE Trans. AC-39:714-724

[16] Sontag, E., 1979 "Realization Theory of Discrete-Time Nonlinear Systems: Part I - the Bounded Case", IEEE Trans. on Circuits and Systems, Vol. 26, No. 4

[17] Sontag, E., Conversations, various times and places, 1994. 